\title{
On thermo-mechanical nonlinear behaviour of shallow shells
}

\author{
P. Khazaeinejad*, A.S. Usmani \\ Institute for Infrastructure and Environment, School of Engineering, The University of \\ Edinburgh, The King's Buildings, Edinburgh EH9 3JL, UK
}

\begin{abstract}
The structural performance of thin shells is largely dictated by their curvature and the degree of lateral restraint at the shell edges. The present study is an attempt to theoretically investigate the influence of such factors on nonlinear thermo-mechanical response of shallow shells with single and double curvatures. For the mechanical loading, a transverse load is assumed and for the thermal loading, a through-depth thermal gradient is applied on the shallow shell. Two types of boundary conditions are considered for the shallow shell, both of which constrain transverse deflections of the shell but allow rotations parallel to the shell boundaries to be free. One of the boundary conditions permits lateral translation (laterally unrestrained) and the other one does not (laterally restrained). The fundamental nonlinear equations of shallow shells are derived based on the quasi-static conditions. The validity and reliability of the proposed approach is assessed by calculating several numerical examples for shallow shells under various mechanical and thermal loads. It is found that the proposed formulation, in particular, can adequately capture the nonlinear behaviour of laterally restrained shallow shells.
\end{abstract}

Keywords: Geometrically nonlinear analysis, Shallow shells, Thermo-mechanical loading, Laterally restrained boundary condition

\footnotetext{
${ }^{*}$ Corresponding author. Tel.: +44 (0) 1316505806.

Email address: p.khazaeinejad@ed.ac.uk (P. Khazaeinejad)
} 


\section{Introduction}

It is well known that shells can deliver useful load-carrying capacity by their curvatures, thereby effectively resisting the external applied loads with optimum use of material. This makes them practical and efficient structural components and of importance in the design of light weight thin-walled structures. Shell components are widely used in buildings (typically as roof structures), bridges, aerospace vehicles, ship hulls, pressure vessels and car bodies. Such components are often subjected to different thermo-mechanical loadings. Research in this area has been often focused towards developing efficient shell elements using numerical methods (e.g. see [1-9]).

However, nonlinear thermo-mechanical behaviour of shells has also been studied using adequately accurate analytical and semi-analytical methods that take into account key features and many complexities of shell problems. Woo and Meguid [10] studied the nonlinear analysis of simply supported (laterally unrestrained) shallow spherical shells with functionally graded material properties subjected to transverse mechanical loads and through-depth temperature fields. The governing equations were established based on the von Kármán theory for large transverse deflections and were solved using series solutions. It was revealed that considering thermo-mechanical coupling effects in the shell formulation can affect the nonlinear response of the shell. Nie [11] presented the nonlinear analysis of an imperfect shallow spherical shell on a Pasternak foundation subjected to uniform loads. The shell was assumed elastically restrained against rotational, transverse and in-plane displacements. The asymptotic iteration method was applied to obtain an analytical expression for the external load and the central deflection of the shell. Numerical results indicated that imperfections cause a drop in the load-bearing capacity of the shell. Heuer and Ziegler [12] studied the thermal snap-through and snap-buckling of symmetrically layered shallow shells with polygonal planforms and laterally restrained boundary conditions (BCs) using a two degrees of freedom model derived from a Ritz-Galerkin approximation. 
Amabili [13] investigated the large amplitude of the response of simply supported doubly curved shallow shells with rectangular planform to static and dynamic loads. He used the Donnell and Novozhilov shell theories retaining in-plane inertia to obtain geometrically nonlinear shell responses. Duc and Van Tung [14] studied the nonlinear response of functionally graded cylindrical panels to uniform lateral pressure and uniform and through-depth temperature gradients by an analytical approach associated with a Galerkin method. Formulation was based on the classical shell theory, considering both the von Kármán-Donnell type of kinematic nonlinearity and initial geometrical imperfection. The numerical results revealed that in-plane restraint and temperature conditions play major roles in dictating the response of the functionally graded cylindrical panels. Recently, the nonlinear buckling behaviour of homogeneous and non-homogeneous orthotropic thin-walled truncated conical shells under axial load was presented by Sofiyev and Kuruoglu [15]. The stability and compatibility equations of the problem were derived using the large deformation theory with the von Kármán-Donnell type of kinematic nonlinearity. It was reported that for long truncated conical shells, the effect of non-homogeneity on the nonlinear axial buckling load is negligible.

Although there is no doubt that numerical methods provide greater flexibility for the analysis of shell structures when compared to analytical methods, research on improving analytical methods is vital when computational effort (mostly in terms of analyst effort) is a concern, or when an alternative approach is required to validate and corroborate numerical results. This is particularly very useful for benchmarking finite element codes developed for thermomechanical simulations of shell elements [16]. Moreover, accurate mathematical models could be used to:

(i) obtain rapid solutions of realistic thermo-mechanical analyses of simple shell structures as part of Monte Carlo methods to account for uncertainty in loads and structural form;

(ii) understand the mathematical underpinnings of engineering concepts such 
as "thermal snap-through" in shallow shells under large displacements;

(iii) provide advanced basis functions for hybrid-type computational approaches.

Motivated by this opportunity, in the present work, a nonlinear mathematical model is developed to analyse the large deflections of shallow shells with various types of curvature under thermo-mechanical loading conditions. The shallow shell is under transverse mechanical loading while being subjected to through-depth thermal gradients. Two types of BCs are considered in the analysis: edges laterally unrestrained in translation, henceforth referred to as the "laterally unrestrained" BC; and edges restrained against translation, henceforth referred to as the "laterally restrained" BC. In both BCs, transverse deflections of the shallow shell are restrained but rotations parallel to the shell boundaries are unrestrained. The compatibility and equilibrium equations are solved for the steady-state problem using appropriate series functions. A notable feature of the proposed approach is its good performance and its relatively rapid convergence for shallow shells with the laterally restrained BC. In the case of shallow shells with the laterally unrestrained BC, this is, however, only achieved for 'extremely shallow shells' (see Figure 1). The reason for poor agreement for this $\mathrm{BC}$ is the significant change in curvature which is not able to be captured by the formulation.

\section{Fundamental theory}

Consider a shallow shell under non-uniform through-depth thermal gradients while it is being subjected to a transverse mechanical load. In line with the assumptions of the shallow shell theory, it is assumed that the rise of the shell is relatively small in comparison to its other dimensions (see Figure 1 which is adapted from Donnell [17] and illustrates schematically the ranges of applicability of various shell theories for modelling cylindrical shells). In the case of large deformations, the strain-displacement relations of a shallow shell with accounting for the stretching of the middle surface of the shell are expressed by 


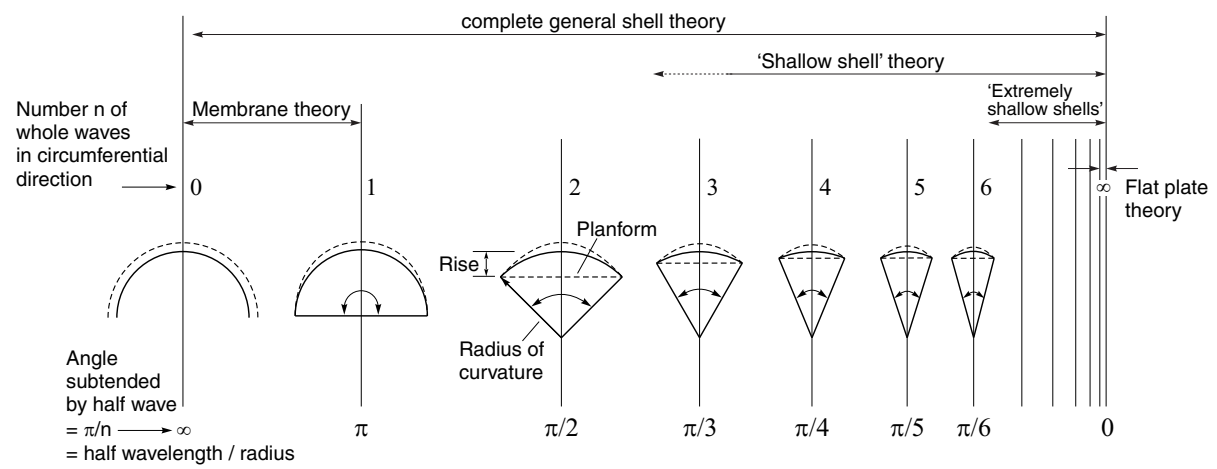

Figure 1: Hierarchy of various shell theories as function of their applicable ranges of subtended angle. The figure is adapted from Donnell [17]. A shallow shell typically has a rise of less than one-fifth of the smallest dimension of its planform. In the case of 'extremely shallow shells', the minimum radius of curvature of the shell is more than two times larger than its maximum planform dimension [18].

$$
\begin{aligned}
\varepsilon_{x x} & =\frac{\partial u}{\partial x}+\frac{1}{2}\left(\frac{\partial w}{\partial x}\right)^{2}+\frac{w}{R_{x}}-z \frac{\partial^{2} w}{\partial x^{2}} \\
\varepsilon_{y y} & =\frac{\partial v}{\partial y}+\frac{1}{2}\left(\frac{\partial w}{\partial y}\right)^{2}+\frac{w}{R_{y}}-z \frac{\partial^{2} w}{\partial y^{2}} \\
\gamma_{x y} & =\frac{\partial u}{\partial y}+\frac{\partial v}{\partial x}+\frac{\partial w}{\partial x} \frac{\partial w}{\partial y}+\frac{2 w}{R_{x y}}-2 z \frac{\partial^{2} w}{\partial x \partial y}
\end{aligned}
$$

where $u, v$ and $w$ are the displacements of the middle surface of the shell in $x$, $y$ and $z$-directions, respectively, $R_{x y}$ represents the twist radius of the middle surface of the shell, and $R_{x}$ and $R_{y}$ are the radii of curvature of the undeformed shell as illustrated in Figure 2 for shallow hyperbolic paraboloidal shell (with double positive and negative curvature), shallow spherical shell (with double curvature) and shallow cylindrical shell (with single curvature). For a shell with thickness of $h$, elastic modulus of $E$, Poisson's ratio of $\nu$, the strain components including thermal effects can be rearranged as

$$
\begin{aligned}
\varepsilon_{x x} & =\frac{1}{E h}\left(N_{x x}-\nu N_{y y}+N^{\theta}\right) \\
\varepsilon_{y y} & =\frac{1}{E h}\left(N_{y y}-\nu N_{x x}+N^{\theta}\right) \\
\gamma_{x y} & =\frac{2(1+\nu)}{E h} N_{x y}
\end{aligned}
$$


The thermal stress resultant $N^{\theta}$ is given by

$$
N^{\theta}=E \alpha \int_{-h / 2}^{h / 2} \theta(z) d z
$$

where $\alpha$ is the coefficient of thermal expansion and $\theta$ describes temperature changes across the shell thickness. The stress resultants can be expressed in terms of a stress function as follows

$$
\begin{aligned}
& N_{x x}=h \frac{\partial^{2} F}{\partial y^{2}} \\
& N_{y y}=h \frac{\partial^{2} F}{\partial x^{2}} \\
& N_{x y}=-h \frac{\partial^{2} F}{\partial x \partial y}
\end{aligned}
$$

It must be noted that the stress function $F$ is an unknown parameter that is determined based on the BCs assumed at the supports of the shallow shell.

\section{Governing equations}

Under quasi-static conditions, the nonlinear response of a thermo-mechanically loaded shell can be determined by solving compatibility and equilibrium equations simultaneously. These are a system of two coupled nonlinear equations. The compatibility equation relates the internal membrane forces caused by large deformations to the transverse displacement of the shell. It can be obtained by

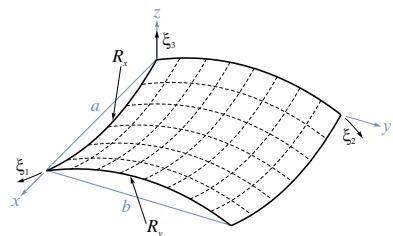

Hyperbolic paraboloidal, $R_{x}=-R$

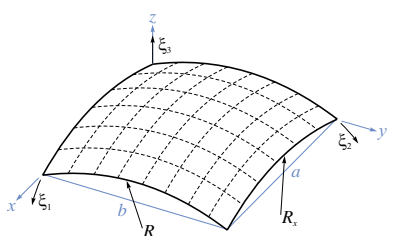

Spherical, $R_{x}=R$

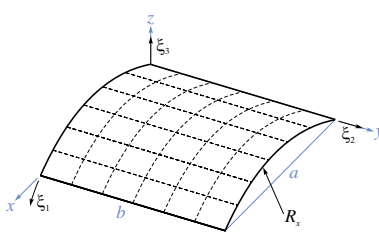

Cylindrical, $R_{y}=\infty$

Figure 2: Various types of curvature for shallow shells with rectangular planform. The curvilinear coordinates on the middle surface of the shells are replaced with a Cartesian coordinate system due to a comparatively small rise above the shells' rectangular planform. It must be noted that for untwisted shallow shells the radius $R_{x y}$ is infinite. 
taking the second derivatives of the strains and combining the resulting expressions as follows:

$\frac{\partial^{2} \varepsilon_{x x}}{\partial y^{2}}+\frac{\partial^{2} \varepsilon_{y y}}{\partial x^{2}}-\frac{\partial^{2} \gamma_{x y}}{\partial x \partial y}=\left(\frac{\partial^{2} w}{\partial x \partial y}\right)^{2}-\frac{\partial^{2} w}{\partial x^{2}} \frac{\partial^{2} w}{\partial y^{2}}+\frac{1}{R_{y}} \frac{\partial^{2} w}{\partial x^{2}}+\frac{1}{R_{x}} \frac{\partial^{2} w}{\partial y^{2}}-\frac{2}{R_{x y}} \frac{\partial^{2} w}{\partial x \partial y}$

Substituting equations (1) and (4) in the above equation, gives us the compatibility equation of the problem to be solved in terms of stress function and transverse deflection as follows:

$$
\begin{aligned}
& \frac{\partial^{4} F}{\partial x^{4}}+2 \frac{\partial^{4} F}{\partial x^{2} \partial y^{2}}+\frac{\partial^{4} F}{\partial y^{4}} \\
& -E\left[\left(\frac{\partial^{2} w}{\partial x \partial y}\right)^{2}-\frac{\partial^{2} w}{\partial x^{2}} \frac{\partial^{2} w}{\partial y^{2}}+\frac{1}{R_{x}} \frac{\partial^{2} w}{\partial y^{2}}+\frac{1}{R_{y}} \frac{\partial^{2} w}{\partial x^{2}}-\frac{2}{R_{x y}} \frac{\partial^{2} w}{\partial x \partial y}\right] \\
& +\frac{1}{h}\left(\frac{\partial^{2} N^{\theta}}{\partial x^{2}}+\frac{\partial^{2} N^{\theta}}{\partial y^{2}}\right)=0
\end{aligned}
$$

The equilibrium equation, however, relates the axial forces to the transverse displacement of the shell as follows

$$
\begin{aligned}
& \frac{\partial^{2} M_{x x}}{\partial x^{2}}+2 \frac{\partial^{2} M_{x y}}{\partial x \partial y}+\frac{\partial^{2} M_{y y}}{\partial y^{2}}+N_{x x} \frac{\partial^{2} w}{\partial x^{2}}+N_{y y} \frac{\partial^{2} w}{\partial y^{2}} \\
& \quad+2 N_{x y} \frac{\partial^{2} w}{\partial x \partial y}-\left(\frac{N_{x x}}{R_{x}}+\frac{N_{y y}}{R_{y}}+\frac{2 N_{x y}}{R_{x y}}\right)+q(x, y)=0
\end{aligned}
$$

where the moment resultants are

$$
\begin{aligned}
& M_{x x}=-D\left(\frac{\partial^{2} w}{\partial x^{2}}+\nu \frac{\partial^{2} w}{\partial y^{2}}\right)-M^{\theta} \\
& M_{y y}=-D\left(\nu \frac{\partial^{2} w}{\partial x^{2}}+\frac{\partial^{2} w}{\partial y^{2}}\right)-M^{\theta} \\
& M_{x y}=-D(1-\nu) \frac{\partial^{2} w}{\partial x \partial y}
\end{aligned}
$$

and the thermal moment resultant $M^{\theta}$ is given by

$$
M^{\theta}=\frac{E \alpha}{1-\nu} \int_{-h / 2}^{h / 2} \theta(z) z d z
$$


After substitution from Eqs. (8a)-(8c) into Eq. (7), the equilibrium equation of the shallow shell can be derived as follows

$$
\begin{aligned}
& D\left(\frac{\partial^{4} w}{\partial x^{4}}+2 \frac{\partial^{4} w}{\partial x^{2} \partial y^{2}}+\frac{\partial^{4} w}{\partial y^{4}}\right)-h\left[\frac{\partial^{2} F}{\partial y^{2}}\left(\frac{\partial^{2} w}{\partial x^{2}}-\frac{1}{R_{x}}\right)\right. \\
& \left.+\frac{\partial^{2} F}{\partial x^{2}}\left(\frac{\partial^{2} w}{\partial y^{2}}-\frac{1}{R_{y}}\right)-2 \frac{\partial^{2} F}{\partial x \partial y}\left(\frac{\partial^{2} w}{\partial x \partial y}-\frac{1}{R_{x y}}\right)\right] \\
& +\left(\frac{\partial^{2} M^{\theta}}{\partial x^{2}}+\frac{\partial^{2} M^{\theta}}{\partial y^{2}}\right)-q(x, y)=0
\end{aligned}
$$

where $D$ is the flexural rigidity of the shell $\left(=E h^{3} / 12\left(1-\nu^{2}\right)\right), q$ is the applied mechanical load. The most common way of solving equations (6) and (10) is to use trigonometric functions. In terms of how the support conditions are specified, appropriate series solutions might be considered.

\section{Solution method}

In this study, two common types of support conditions are considered for the shallow shell:

1. Edges laterally unrestrained thereby permitting lateral translation, while transverse translations are restrained along the shell edges and rotations about the edge axes are free.

2. Edges laterally restrained where all translations are restricted along the shell edges while the rotation about the edge axes remains free.

These BCs are reasonable limiting cases bracketing the conditions that may be found in real shell structures (except for cases where rotational restraints are important). The transverse displacement can be expanded in a double Fourier series as follows:

$$
w(x, y)=\sum_{m=1}^{\infty} \sum_{n=1}^{\infty} w_{m n} S_{m n}
$$

where $S_{m n}=\sin (m \pi x / a) \sin (n \pi y / b)$. The thermal resultants may also be taken in the following forms:

$$
\left\{\begin{array}{l}
N^{\theta}(x, y) \\
M^{\theta}(x, y)
\end{array}\right\}=\sum_{m=1}^{\infty} \sum_{n=1}^{\infty}\left\{\begin{array}{c}
N_{m n}^{\theta} \\
M_{m n}^{\theta}
\end{array}\right\} S_{m n}
$$


where the coefficients are calculated by performing the Fourier integration as follows:

$$
\left\{\begin{array}{l}
N_{m n}^{\theta} \\
M_{m n}^{\theta}
\end{array}\right\}=\frac{4\left[-1+(-1)^{m}\right]\left[-1+(-1)^{n}\right]}{m n \pi^{2}}\left\{\begin{array}{l}
N^{\theta} \\
M^{\theta}
\end{array}\right\}
$$

In a similar way, different transverse mechanical loading conditions can be considered by expressing the traction $q$ in a double Fourier series as

$$
q(x, y)=\sum_{m=1}^{\infty} \sum_{n=1}^{\infty} q_{m n} S_{m n}
$$

where the coefficient of the series is given by

$$
q_{m n}=\frac{4}{a b} \int_{0}^{a} \int_{0}^{b} q(x, y) \sin \frac{m \pi x}{a} \sin \frac{n \pi y}{b} d y d x
$$

The following loading conditions are then obtained:

For uniform loading of magnitude $q$ :

$$
q_{m n}=\frac{4\left[-1+(-1)^{m}\right]\left[-1+(-1)^{n}\right]}{m n \pi^{2}} q
$$

For sinusoidal loading of magnitude $q$ where $m=n=1$ :

$$
q_{m n}=q
$$

For point load of $q$ applied at coordinates $\left(x_{0}, y_{0}\right)$ :

$$
q_{m n}=\frac{4 q_{0}}{a b} \sin \frac{m \pi x_{0}}{a} \sin \frac{n \pi y_{0}}{b}
$$

For the Airy stress function, an expression satisfying the stress-free edges case (for the laterally unrestrained $\mathrm{BC}$ ) and undeformed edges case (for the laterally restrained $\mathrm{BC}$ ) may then be taken as

$$
F(x, y)=\frac{P_{x} y^{2}}{2 b h}+\frac{P_{y} x^{2}}{2 a h}+\sum_{m=1}^{\infty} \sum_{n=1}^{\infty} F_{m n} S_{m n}
$$

where $P_{x}$ and $P_{y}$ are the total loads applied on the sides $x=0, a$ and $y=$ $0, b$, respectively. For the laterally unrestrained BC, such loads are zero. In contrast, if the support conditions corresponding to the laterally restrained $\mathrm{BC}$ 
are imposed, then the following expressions can be obtained from the elongation of the shell in the $x$ direction

$$
\int_{0}^{a} \frac{\partial u}{\partial x} d x=\int_{0}^{a}\left[\frac{1}{E}\left(\frac{\partial^{2} F}{\partial y^{2}}-\nu \frac{\partial^{2} F}{\partial x^{2}}\right)-\frac{1}{2}\left(\frac{\partial w}{\partial x}\right)^{2}-\frac{w}{R_{x}}+\frac{N^{\theta}}{E h}\right] d x
$$

and in the $y$ direction

$$
\int_{0}^{b} \frac{\partial v}{\partial y} d y=\int_{0}^{b}\left[\frac{1}{E}\left(\frac{\partial^{2} F}{\partial x^{2}}-\nu \frac{\partial^{2} F}{\partial y^{2}}\right)-\frac{1}{2}\left(\frac{\partial w}{\partial y}\right)^{2}-\frac{w}{R_{y}}+\frac{N^{\theta}}{E h}\right] d y
$$

where setting the edge displacements to zero after substituting Eqs. (11) and (19) into Eqs. (20) and (21), and performing the integration, yields the following expressions for $P_{x}$

$$
\begin{aligned}
P_{x}= & \frac{E b h \pi^{2} w_{m n}^{2}}{8\left(1-\nu^{2}\right)}\left(\frac{m^{2}}{a^{2}}+\nu \frac{n^{2}}{b^{2}}\right)+\frac{h n\left[-1+(-1)^{m}\right]\left[-1+(-1)^{n}\right] F_{m n}}{m b} \\
& +\frac{E a h\left[-1+(-1)^{m}\right]\left[-1+(-1)^{n}\right] w_{m n}}{m n \pi^{2}\left(1-\nu^{2}\right)}\left(\frac{1}{R_{x}}+\frac{\nu}{R_{y}}\right)-\frac{b N^{\theta}}{(1-\nu)}
\end{aligned}
$$

and $P_{y}$

$$
\begin{aligned}
P_{y}= & \frac{E a h \pi^{2} w_{m n}^{2}}{8\left(1-\nu^{2}\right)}\left(\nu \frac{m^{2}}{a^{2}}+\frac{n^{2}}{b^{2}}\right)+\frac{h m\left[-1+(-1)^{m}\right]\left[-1+(-1)^{n}\right] F_{m n}}{n a} \\
& +\frac{E a h\left[-1+(-1)^{m}\right]\left[-1+(-1)^{n}\right] w_{m n}}{m n \pi^{2}\left(1-\nu^{2}\right)}\left(\frac{\nu}{R_{x}}+\frac{1}{R_{y}}\right)-\frac{a N^{\theta}}{(1-\nu)}
\end{aligned}
$$

By taking account of the mathematical coupling between higher order terms in the governing equations more than two indices must be used for representing the series solution terms. Substituting Eqs. (11)-(19) into Eqs. (6) and (10) leads to the the following compatibility equation

$$
\begin{aligned}
& \left(\frac{m^{2}}{a^{2}}+\frac{n^{2}}{b^{2}}\right)^{2} F_{m n} S_{m n}-\left(\frac{m^{2}}{a^{2}}+\frac{n^{2}}{b^{2}}\right) \frac{N_{m n}^{\theta} S_{m n}}{h \pi^{2}} \\
& -\frac{E}{a^{2} b^{2}}\left(m n r s C_{m n} C_{r s}-m^{2} s^{2} S_{m n} S_{r s}\right) w_{m n} w_{r s} \\
& +\frac{E}{\pi^{2}}\left(\frac{n^{2} S_{m n}}{b^{2}} \frac{1}{R_{x}}+\frac{m^{2} S_{m n}}{a^{2}} \frac{1}{R_{y}}+\frac{m n C_{m n}}{a b} \frac{2}{R_{x y}}\right) w_{m n}=0
\end{aligned}
$$


and equilibrium equation

$$
\begin{aligned}
& {\left[D\left(\frac{m^{2}}{a^{2}}+\frac{n^{2}}{b^{2}}\right)^{2}+\frac{1}{a b \pi^{2}}\left(\frac{m^{2} P_{x}}{a}+\frac{n^{2} P_{y}}{b}\right)\right] w_{m n} S_{m n}} \\
& -\left(\frac{m^{2}}{a^{2}}+\frac{n^{2}}{b^{2}}\right) \frac{M_{m n}^{\theta} S_{m n}}{\pi^{2}} \\
& \quad-\frac{h\left[\left(m^{2} s^{2}+r^{2} n^{2}\right) S_{m n} S_{r s}-2 m r n s C_{m n} C_{r s}\right]}{a^{2} b^{2}} F_{r s} w_{m n} \\
& -\frac{h}{\pi^{4}}\left[\left(-\frac{P_{x}}{b h}+\frac{s^{2} \pi^{2}}{b^{2}} F_{r s} S_{r s}\right) \frac{1}{R_{x}}+\left(-\frac{P_{y}}{a h}+\frac{r^{2} \pi^{2}}{a^{2}} F_{r s} S_{r s}\right) \frac{1}{R_{y}}\right. \\
& \left.+\frac{2 r s \pi^{2} C_{r s}}{a b} F_{r s} \frac{1}{R_{x y}}\right]-\frac{q_{m n} S_{m n}}{\pi^{4}}=0
\end{aligned}
$$

where $C_{m n}=\cos (m \pi x / a) \cos (n \pi y / b)$. As can be seen, considering the mathematical coupling raises the computational time needed to solve the derived system of equations. Moreover, its contribution to the final solution might be negligible as it has been shown for flat shells in Khazaeinejad et al. [19]. Hence, ignoring the mathematical coupling (i.e., $m=r$ and $n=s$ ) results in a simplified version of the compatibility equation

$$
\begin{aligned}
& \left(\frac{m^{2}}{a^{2}}+\frac{n^{2}}{b^{2}}\right)^{2} F_{m n} S_{m n}-\left(\frac{m^{2}}{a^{2}}+\frac{n^{2}}{b^{2}}\right) \frac{N_{m n}^{\theta} S_{m n}}{h \pi^{2}} \\
& -\frac{E m^{2} n^{2}\left(C_{m n}^{2}-S_{m n}^{2}\right)}{a^{2} b^{2}} w_{m n}^{2} \\
& +\frac{E}{\pi^{2}}\left(\frac{n^{2} S_{m n}}{b^{2}} \frac{1}{R_{x}}+\frac{m^{2} S_{m n}}{a^{2}} \frac{1}{R_{y}}+\frac{m n C_{m n}}{a b} \frac{2}{R_{x y}}\right) w_{m n}=0
\end{aligned}
$$

and the equilibrium equation

$$
\begin{aligned}
& {\left[D\left(\frac{m^{2}}{a^{2}}+\frac{n^{2}}{b^{2}}\right)^{2}+\frac{1}{a b \pi^{2}}\left(\frac{m^{2} P_{x}}{a}+\frac{n^{2} P_{y}}{b}\right)\right] w_{m n} S_{m n}} \\
& -\left(\frac{m^{2}}{a^{2}}+\frac{n^{2}}{b^{2}}\right) \frac{M_{m n}^{\theta} S_{m n}}{\pi^{2}}-\frac{2 h m^{2} n^{2}\left(S_{m n}^{2}-C_{m n}^{2}\right)}{a^{2} b^{2}} F_{m n} w_{m n} \\
& -\frac{h}{\pi^{4}}\left[\left(-\frac{P_{x}}{b h}+\frac{n^{2} \pi^{2}}{b^{2}} F_{m n} S_{m n}\right) \frac{1}{R_{x}}\right. \\
& \left.+\left(-\frac{P_{y}}{a h}+\frac{m^{2} \pi^{2}}{a^{2}} F_{m n} S_{m n}\right) \frac{1}{R_{y}}+\frac{2 m n \pi^{2} C_{m n}}{a b} F_{m n} \frac{1}{R_{x y}}\right] \\
& -\frac{q_{m n} S_{m n}}{\pi^{4}}=0
\end{aligned}
$$


It should be noted that in the case of untwisted shallow shells, the twist radius $R_{x y}$ should be infinite and therefore the whole terms containing $1 / R_{x y}$ vanish. Using the expansion theorem [20], if the left part of the equation (26) is denoted by $X_{1}$ and of the equation (27) by $X_{2}$, then from the following equations

$$
\int_{0}^{a} \int_{0}^{b}\left\{\begin{array}{c}
X_{1} \\
X_{2}
\end{array}\right\} S_{m n}=0
$$

the following algebraic equations can be derived:

$$
\begin{gathered}
\left(\frac{m^{2}}{a^{2}}+\frac{n^{2}}{b^{2}}\right)^{2} F_{m n}-\left(\frac{m^{2}}{a^{2}}+\frac{n^{2}}{b^{2}}\right) \frac{N_{m n}^{\theta}}{h \pi^{2}} \\
-4 E H_{m n}\left(\frac{m n}{a b}\right)^{2} w_{m n}^{2}+\frac{E}{\pi^{2}}\left(\frac{n^{2}}{b^{2}} \frac{1}{R_{x}}+\frac{m^{2}}{a^{2}} \frac{1}{R_{y}}\right) w_{m n}=0 \\
\quad\left[D\left(\frac{m^{2}}{a^{2}}+\frac{n^{2}}{b^{2}}\right)^{2}+\frac{1}{a b \pi^{2}}\left(\frac{m^{2} P_{x}}{a}+\frac{n^{2} P_{y}}{b}\right)\right] w_{m n} \\
-\left(\frac{m^{2}}{a^{2}}+\frac{n^{2}}{b^{2}}\right) \frac{M_{m n}^{\theta}}{\pi^{2}}+8 h H_{m n} F_{m n}\left(\frac{m n}{a b}\right)^{2} w_{m n} \\
+\frac{4\left[-1+(-1)^{m}\right]\left[-1+(-1)^{n}\right]}{m n \pi^{6}}\left(\frac{P_{x}}{b} \frac{1}{R_{x}}+\frac{P_{y}}{a} \frac{1}{R_{y}}\right) \\
\quad-\frac{h}{\pi^{2}}\left(\frac{n^{2}}{b^{2}} \frac{1}{R_{x}}+\frac{m^{2}}{a^{2}} \frac{1}{R_{y}}\right) F_{m n}-\frac{q_{m n}}{\pi^{4}}=0
\end{gathered}
$$

where

$H_{m n}=\frac{-1+2(-1)^{m}+2(-1)^{n}-(-1)^{3 m}-(-1)^{3 n}-3(-1)^{m+n}+(-1)^{3 m+n}+(-1)^{m+3 n}}{3 m n \pi^{2}}$

Substituting Eq. (29) into Eq. (30), gives the following characteristic loaddeflection equation for the shell

$$
\varsigma_{1} w_{m n}^{3}+\varsigma_{2} w_{m n}^{2}+\varsigma_{3} w_{m n}+\varsigma_{4}=0
$$

where

$$
\begin{aligned}
& \varsigma_{1}=\frac{32 E h m^{4} n^{4} H_{m n}^{2}}{\left(m^{2} b^{2}+n^{2} a^{2}\right)^{2}} \\
& \varsigma_{2}=-\frac{12 E h H_{m n} a^{2} b^{2}}{\pi^{2}\left(m^{2} b^{2}+n^{2} a^{2}\right)^{2}}\left(\frac{m^{2} n^{4}}{b^{2}} \frac{1}{R_{x}}+\frac{m^{4} n^{2}}{a^{2}} \frac{1}{R_{y}}\right)
\end{aligned}
$$




$$
\begin{aligned}
\varsigma_{3}= & D\left(\frac{m^{2}}{a^{2}}+\frac{n^{2}}{b^{2}}\right)^{2}+\frac{8 N_{m n}^{\theta} H_{m n} m^{2} n^{2}}{\pi^{2}\left(m^{2} b^{2}+n^{2} a^{2}\right)}+\frac{1}{a b \pi^{2}}\left(\frac{m^{2} P_{x}}{a}+\frac{n^{2} P_{y}}{b}\right) \\
& +\frac{E h}{\pi^{4}\left(m^{2} b^{2}+n^{2} a^{2}\right)^{2}}\left(n^{2} a^{2} \frac{1}{R_{x}}+m^{2} b^{2} \frac{1}{R_{y}}\right)^{2} \\
\varsigma_{4}= & -\left(\frac{m^{2}}{a^{2}}+\frac{n^{2}}{b^{2}}\right) \frac{M_{m n}^{\theta}}{\pi^{2}}-\frac{N_{m n}^{\theta} a^{2} b^{2}}{\pi^{4}\left(m^{2} b^{2}+n^{2} a^{2}\right)}\left(\frac{n^{2}}{b^{2}} \frac{1}{R_{x}}+\frac{m^{2}}{a^{2}} \frac{1}{R_{y}}\right) \\
& -\frac{q_{m n}}{\pi^{4}}+\frac{4\left[-1+(-1)^{m}\right]\left[-1+(-1)^{n}\right]}{m n \pi^{6}}\left(\frac{P_{x}}{b} \frac{1}{R_{x}}+\frac{P_{y}}{a} \frac{1}{R_{y}}\right)
\end{aligned}
$$

There are three solutions for the equation 32 , one real solution and two complex solutions. The deflection must be calculated for adequate series terms to make the final solution more accurate for both BCs. The membrane tractions of the shell can then be calculated from

$$
\begin{gathered}
N_{x x}=\frac{P_{x}}{b}-\sum_{m=1}^{\infty} \sum_{n=1}^{\infty} \frac{h n^{2} \pi^{2}}{b^{2}} S_{m n} F_{m n} \\
N_{y y}=\frac{P_{y}}{a}-\sum_{m=1}^{\infty} \sum_{n=1}^{\infty} \frac{h m^{2} \pi^{2}}{a^{2}} S_{m n} F_{m n} \\
N_{x y}=-\sum_{m=1}^{\infty} \sum_{n=1}^{\infty} \frac{h m n \pi^{2}}{a b} C_{m n} F_{m n}
\end{gathered}
$$

It should be noted that in order to quantify the evolution of the membrane action in the shallow shells, a limited number of terms should be retained in the above series.

\section{Results and discussion}

The good accuracy of the proposed method for flat shells under different thermo-mechanical actions has already been confirmed [20]. To investigate its accuracy and performance for shallow shells, a number of examples are solved in the following for square shallow shells with both the chosen BCs. In the examples considered, the Poisson's ratio is equal to 0.3 and the shells are subjected to a UDL and/or through-depth thermal gradients. The dimensionless quantities used are listed in Table 1. 
First, the results of dimensionless deflection for a shallow spherical shell with the laterally unrestrained $\mathrm{BC}$ under thermal loading are compared with results obtained from a meshless local Petrov-Galerkin method (MLPG) formulation [21] and a finite element method (FEM) analysis [21]. The results are plotted in Figure 3. The shell has elastic modulus of $0.6895 \times 10^{10} \mathrm{~N} / \mathrm{m}^{2}$, coefficient of thermal expansion of $1 \times 10^{-5} 1 /{ }^{\circ} \mathrm{C}$, thickness to span ratio of 0.05 , and radius of curvature to span ratio of 10 . A uniformly distributed temperature of $1^{\circ} \mathrm{C}$ is applied at the top surface of the shell, while the temperature at the bottom surface of the shell is zero. It can be seen that the solutions of the proposed method are in close agreement with the solutions obtained from both the MLPG method and FEM with a rather rapid convergence (with considering only one series term in the solutions).

The results of dimensionless deflection of a shallow spherical shell with the laterally unrestrained BC subjected to a UDL are also compared with the results obtained from the MLPG formulation [5], as shown in Figure 4a. The bottom surface of the shell is subjected to a UDL of $2.07 \times 10^{6} \mathrm{~N} / \mathrm{m}^{2}$ directed upwards (bottom surface loading). The shell has the same material and geometrical properties as the previous example. Only for this example, the deflection is presented in a different dimensionless form, dividing the deflection of shallow shell by the deflection of its corresponding flat shell $\left(R_{x}=R_{y}=\infty\right)$. Figure 4a shows that the maximum deflection at the centre of the laterally unrestrained

Table 1: Definitions of dimensionless quantities

\begin{tabular}{ll}
\hline Description & Definition \\
\hline Thickness to span ratio & $h / a$ \\
Rise to thickness ratio & $H / h$ \\
Dimensionless UDL & $q a^{4} / E h^{4}$ \\
Radius of curvature to span ratio & $R_{x} / a$ and $R_{y} / a$ \\
Dimensionless deflection & $w / h$ \\
Dimensionless $x$-coordinate parameter & $x / a$ \\
\hline
\end{tabular}




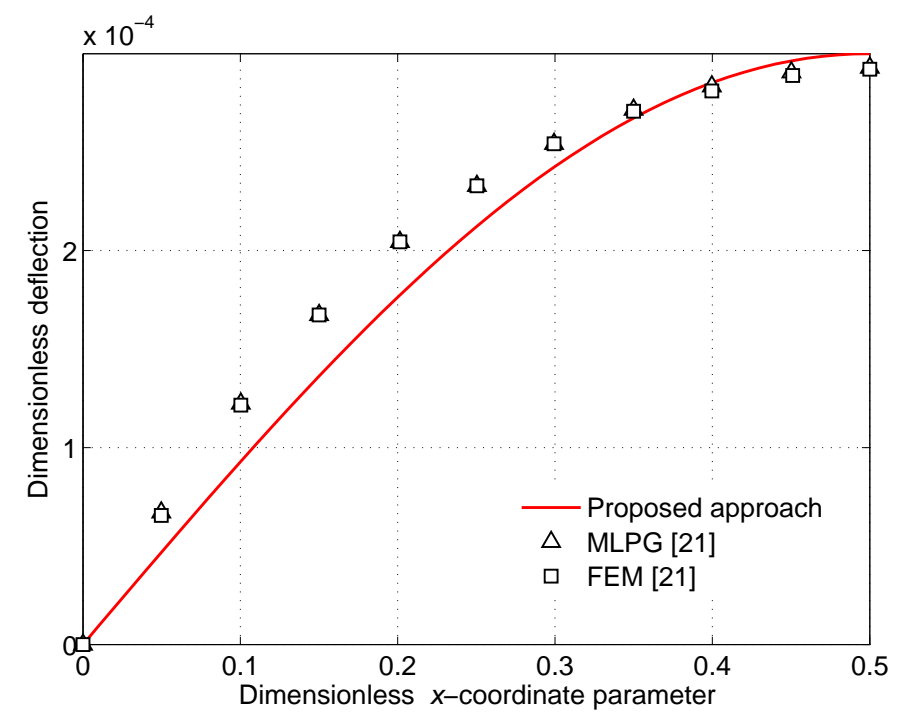

Figure 3: Variation of dimensionless deflection with the dimensionless $x$-coordinate parameter for a shallow spherical shell with the laterally unrestrained BC subject to thermal loading.

shallow shell differ by 20 percent from the deflection of its corresponding flat shell. The convergence of the solution for the central deflection is demonstrated in Figure 4b.

As can be seen, the present solutions (for both thermal and mechanical loading) match well with those solutions obtained from the MLPG method. It may also be noticed that this reasonable accuracy may be achieved by considering only one series term in the solutions.

As stated earlier, the curvature allows a thin shell to deliver useful loadcarrying capacity. To better understand this feature, the influence of the curvature of a shallow cylindrical shell (with elastic modulus of $27 \times 10^{9} \mathrm{~N} / \mathrm{m}^{2}$ and thickness to span ratio of 0.03 ) on its nonlinear response to a UDL is investigated using the proposed model and the finite element software ABAQUS. This is shown in Tables 2 and 3, for shallow cylindrical shells subjected to a UDL of 10 directed downwards (top surface loading) with the laterally unrestrained and restrained BCs, respectively. The shell is modelled in ABAQUS using 1764 S4R5 elements. The rise of the shell above its planform is denoted by $H$ and is 


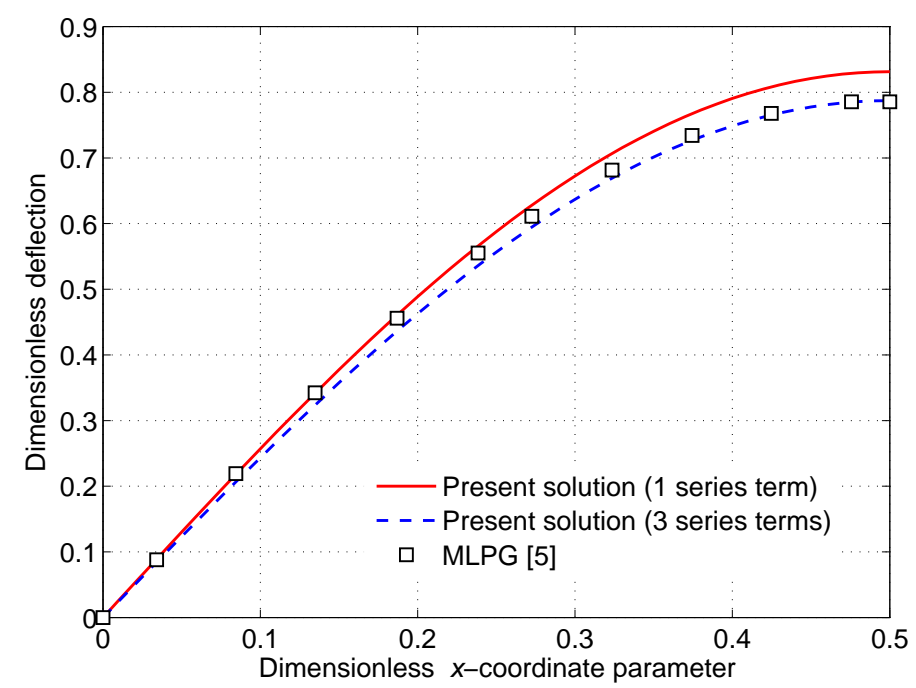

(a)

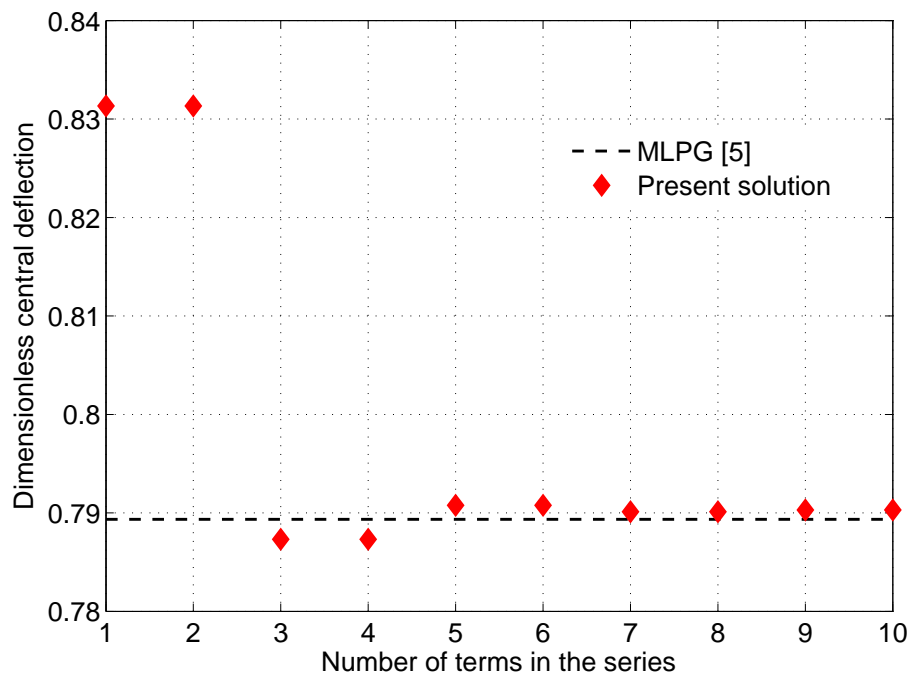

(b)

Figure 4: Comparison of deflection for a shallow spherical shell with the laterally unrestrained BC subjected to UDL. Figure (a) shows the variation of dimensionless deflection (i.e. deflection of the shallow shell over deflection of its corresponding flat shell) with the dimensionless $x$-coordinate parameter and Figure (b) demonstrates the convergence of the dimensionless central deflection. Note that, the denominator of the MLPG and the proposed solutions are determined from the same method used for the calculation of the deflection of the curved shell. 
Table 2: Comparison of dimensionless nonlinear central deflection for a shallow cylindrical shell with the laterally unrestrained BC under UDL using one series term

\begin{tabular}{llll}
\hline Radius of & Rise to Angle subtended & & $\begin{array}{l}\text { Proposed } \\
\text { approach }^{b}\end{array}$ \\
curvature & thickness & ABAQUS simulation \\
to span & ratio & & \\
ratio & & & \\
\hline
\end{tabular}

1

4.47

60

$-0.053$

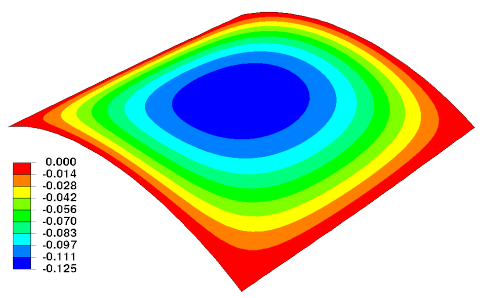

2

2.12

28.95

$-0.172$

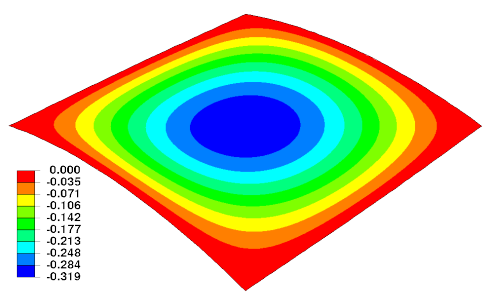

3

$1.40 \quad 19.2$

$-0.297$

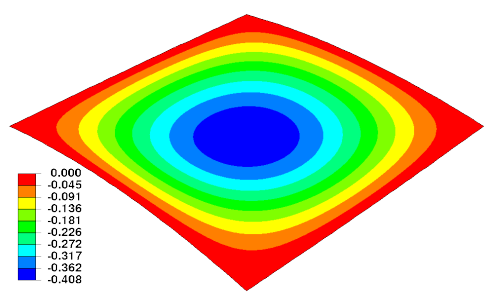

(1)

$a_{\text {See Figure } 1 .}$

${ }^{b}$ The present formulation is only valid for extremely shallow shells (e.g. $R / a=5$ ) with the laterally unrestrained BC. 
Table 3: Comparison of dimensionless nonlinear central deflection for a shallow cylindrical shell with the laterally restrained BC under UDL using three series terms

\begin{tabular}{llll}
\hline Radius of & Rise to Angle subtended $^{a}$ & $\begin{array}{l}\text { Proposed } \\
\text { proach }\end{array}$ & \\
curvature & thickness & ABAQUS simulation \\
to span & ratio & & \\
ratio & & & \\
\hline
\end{tabular}

1

4.47

60

2.12

2

3

1.40

19.2

11.5

5

0.83

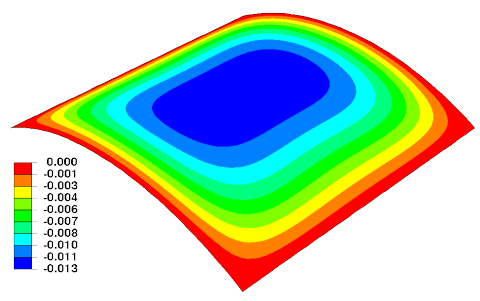

$-0.010$
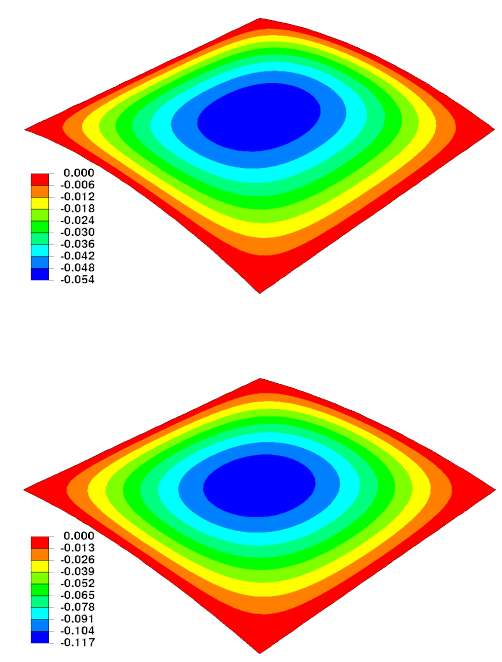

$-0.122$

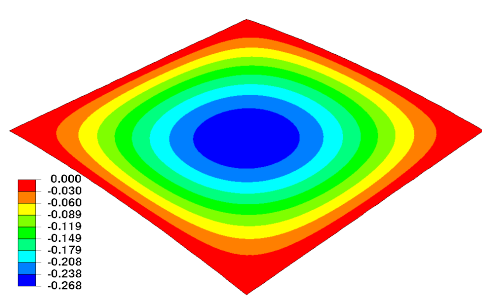

$-0.278$

${ }^{a}$ See Figure 1. 
calculated from $R-\sqrt{R^{2}-a^{2} / 4}$, as illustrated in Figure 1. It can be observed that the deflection values decrease with an increase in the curvature of the shell. This is due to increasing flexural rigidity of the shell caused by the curvature. To justify this, in Figure 5, error in the proposed solutions with respect to the ABAQUS solutions are plotted against the mean squared shell curvature over the plate. As can be seen, the error in both the unrestrained and restrained BCs are increased by an increase in the shell curvature.

The accuracy of the proposed method decreases significantly for relatively deep shells with the laterally unrestrained BC. Therefore, the present approach is not accurate enough for determining the nonlinear responses of such shells. However, in the case of shells with the laterally restrained BC, the proposed method has a very good performance when compared with the ABAQUS solutions. This is very promising since the laterally restrained BC is practically more useful than the laterally unrestrained BC.

The load-deflection relationship for various types of shallow shells (i.e. hyperbolic paraboloidal, spherical, and cylindrical) is depicted in Figures 6 and 7 for both the chosen BCs. The shell has the same material properties as the previous example and is subjected to a UDL at its either top or bottom surface. For both BCs, the maximum deflection of the shell (at the centre) increases nonlinearly as the UDL increases. It is clear that the top surface loading produces a downwards deflection whereas the bottom surface loading produces an upwards deflection, according to the sign convention in Figure 2. It is also interesting to note how the nonlinear behaviour of shallow shells changes due to a change in the radius of the shell curvature. As can be seen in Figures 6 and 7, under both upwardly and downwardly directed mechanical loading, the shallow spherical shell is deflected less than the other two types of shallow shells. As such, the largest deflection has been produced by the shallow hyperbolic paraboloidal and cylindrical shells, depending upon the intensity of the UDL applied. For instance, under downwardly directed UDL of 20, the shallow cylindrical shell has produced the largest deflection while under the same UDL when upwardly directed, the shallow hyperbolic paraboloidal shell has produced the 


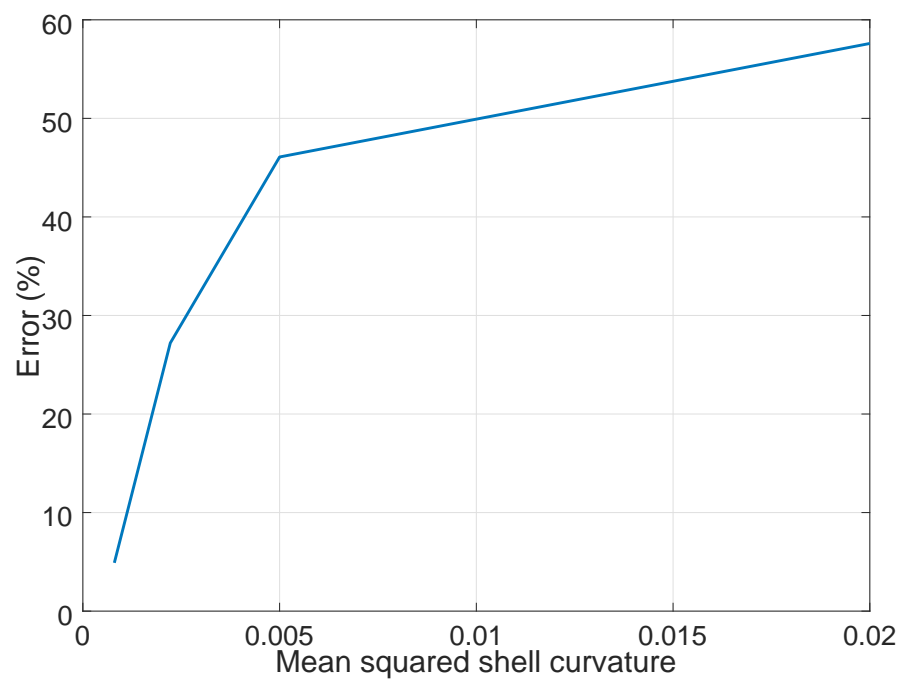

(a)

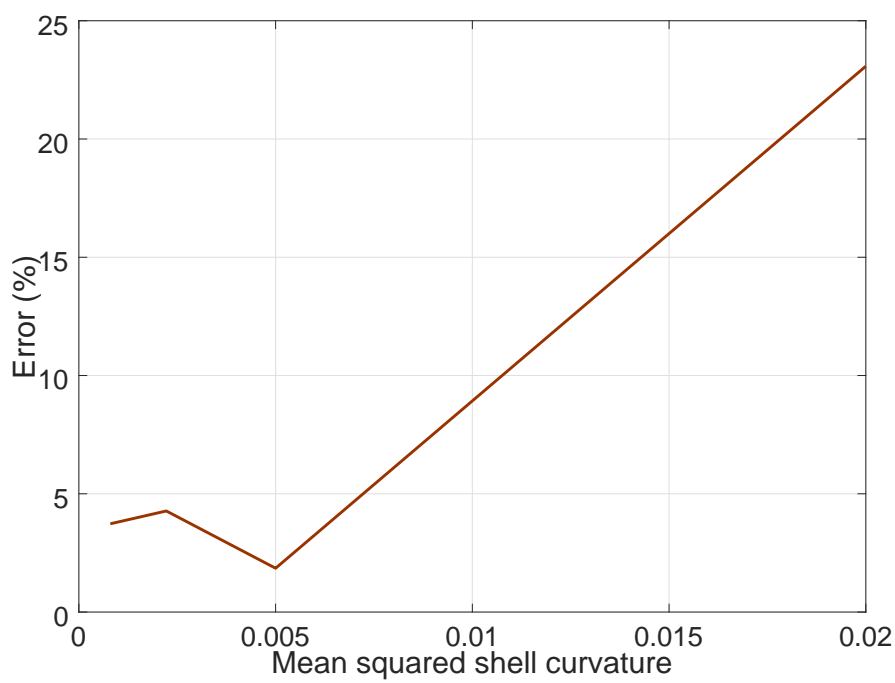

(b)

Figure 5: Variations of the mean squared shell curvature over the plate against the error in the central nonlinear deflection (with respect to the ABAQUS solutions) for a cylindrical shallow shell under UDL; (a) laterally unrestrained BC and (b) laterally restrained BC. 
largest deflection.

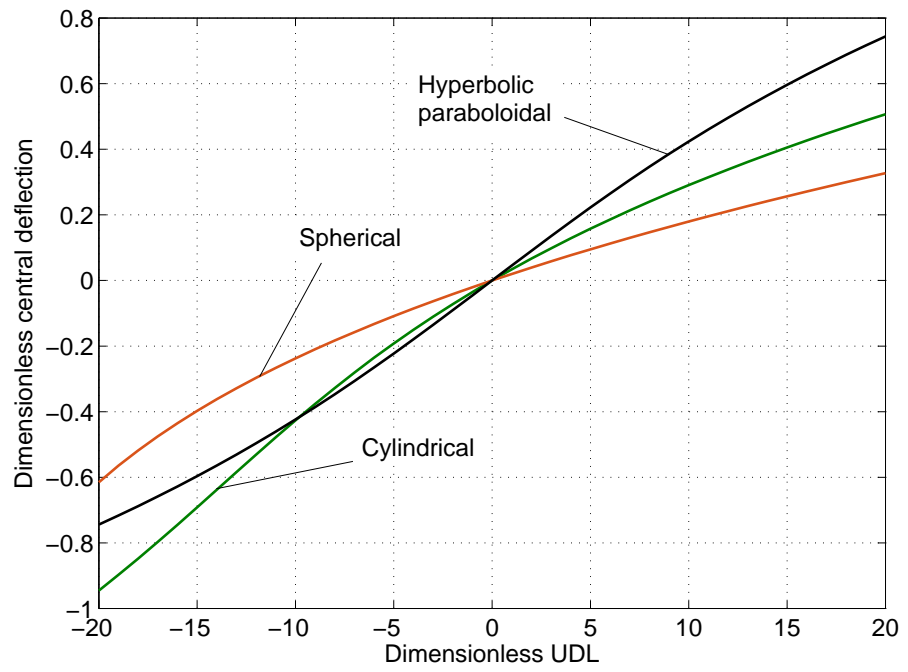

Figure 6: Dimensionless central deflection for various types of shallow shells with the laterally unrestrained BC subjected to UDL. The data are calculated using only one series term.

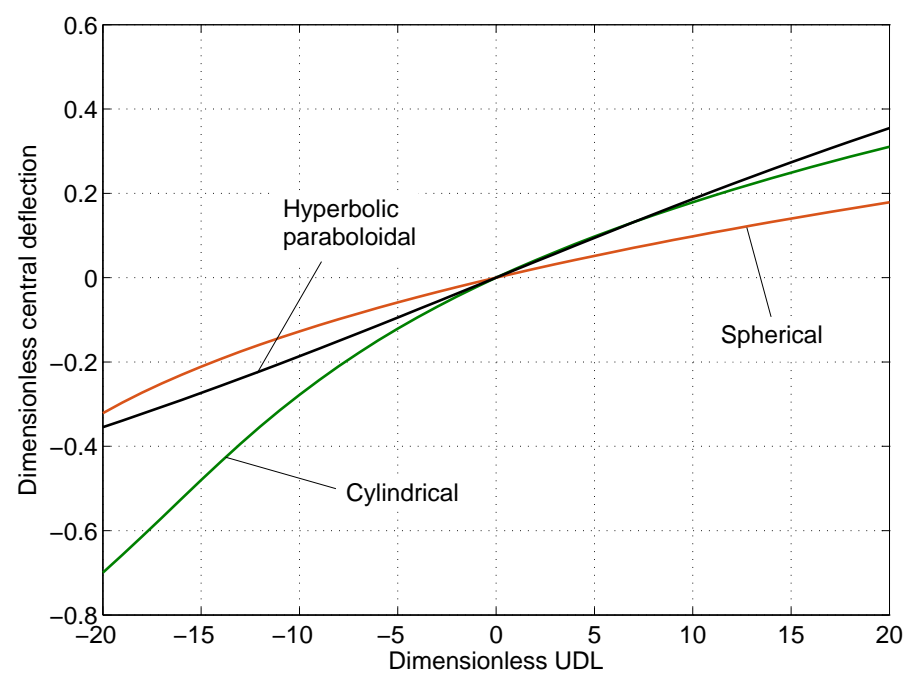

Figure 7: Dimensionless central deflection for various types of shallow shells with the laterally restrained BC subjected to UDL. The data are calculated using three series terms. 
As another example, the central deflection of a shallow hyperbolic paraboloidal shell (i.e $R_{x} / a=-R_{y} / a=-5$ ) subjected to UDL and linear thermal gradient is considered. The temperature at the top surface of the shell was assumed to be the ambient temperature $\left(20^{\circ} \mathrm{C}\right)$ and it assumed to vary linearly through the depth of the shell to temperatures of $20^{\circ} \mathrm{C}, 100^{\circ} \mathrm{C}$, and $200^{\circ} \mathrm{C}$ at the bottom surface. Such temperature profiles may also be represented by an equivalent thermal gradient of $\Delta \theta / z$ and an equivalent mean temperature of $\Delta \theta$. The coefficient of thermal expansion of the shell is taken as $8 \times 10^{-6} 1 /{ }^{\circ} \mathrm{C}$. Other material and geometrical properties are the same as those stated in the previous example. The dimensionless maximum deflection of the shell is depicted in Figures 8 and 9 for both the chosen BCs. The figures show that for the cases where a downwardly directed UDL is applied at the top surface of the shell, larger temperature gradients result in larger deflections. However, the opposite behaviour is seen where an upwardly directed UDL is applied at the bottom surface of the shell. Moreover, as expected, adding lateral restraint at the shell edges has resulted in lower values for the shell maximum deflections as compared with those obtained for the laterally unrestrained shells.

\section{Conclusions}

A theoretical model was developed for the geometrically nonlinear analysis of shallow shells in rectangular planforms subjected to thermo-mechanical loadings. Two types of support conditions were assumed, bracketing reasonable conditions that may be found in real shell structures applications. It was found that using three terms in the series solutions gives results that are reasonably accurate for laterally restrained shallow shells. However, for a shallow shell that is laterally unrestrained, only one term in the series solutions might be adequate, but the solutions were only accurate for extremely shallow shells with a comparatively small rise above their rectangular planform. The reason for this could be the curvature change with respect to the unrestrained boundaries which was related to the structure moving from larger to smaller curvature. Nevertheless, 


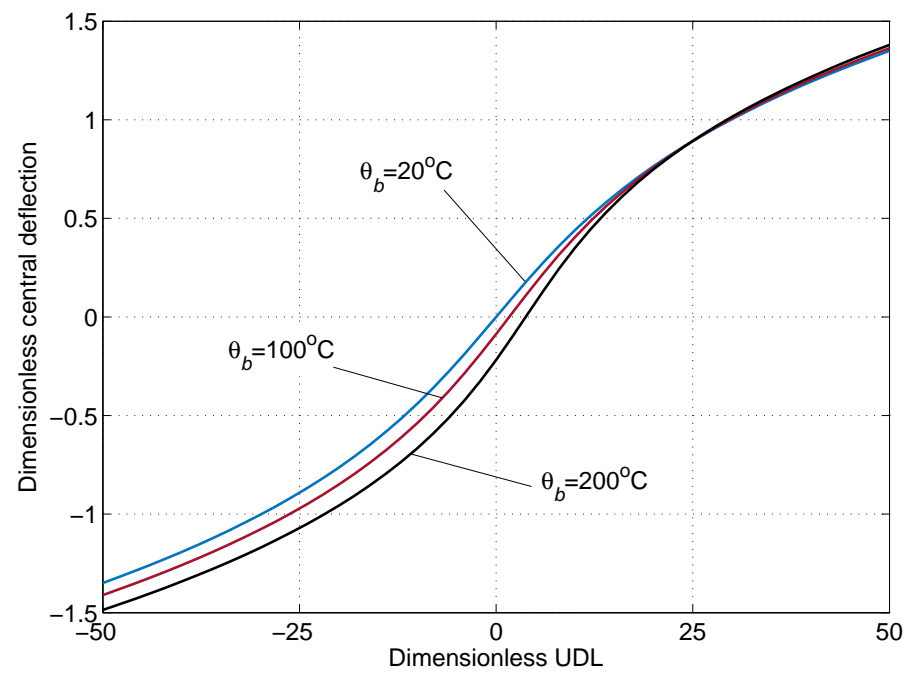

Figure 8: Dimensionless central deflection for a shallow hyperbolic paraboloidal shell with the laterally unrestrained $\mathrm{BC}$ subjected to UDL and linear temperature gradient. The data are calculated using one series term.

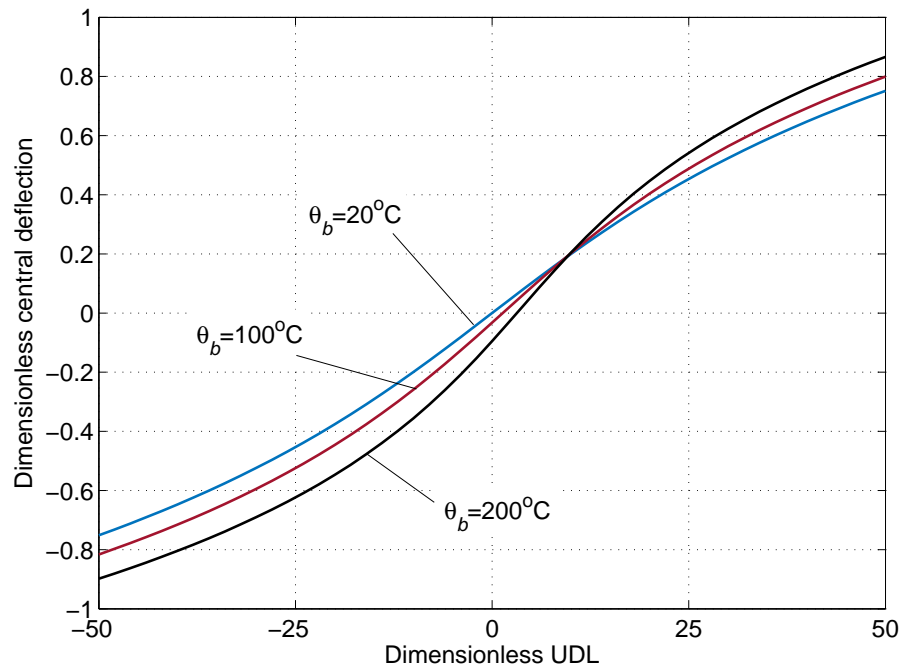

Figure 9: Dimensionless central deflection for a shallow hyperbolic paraboloidal shell with the laterally restrained BC subjected to UDL and linear temperature gradient. The data are calculated using three series terms. 
the model may be applied to snap-through problems. There is no doubt that the change of curvature is greater during snap-through as there is reversal of curvature, however the phenomena can still be captured as just before and just after snap-through the absolute value of the curvatures is very small and within the range where the model has good predictive capability.

\section{Acknowledgement}

The authors are grateful for the financial support for this research through the Edinburgh Research Partnership in Engineering (ERPE) funding.

\section{References}

[1] K.-J. Bathe, P.-S. Lee, J.-F. Hiller, Towards improving the MITC9 shell element, Computers \& Structures 81 (2003) 477-489.

[2] Y. Zhang, K. Kim, Linear and geometrically nonlinear analysis of plates and shells by a new refined non-conforming triangular plate/shell element, Computational Mechanics 36 (2005) 331-342.

[3] M. Brunet, F. Sabourin, Analysis of a rotation-free 4-node shell element, International Journal for Numerical Methods in Engineering 66 (2006) 14831510.

[4] N. Nguyen-Thanh, T. Rabczuk, H. Nguyen-Xuan, S. Bordas, A smoothed finite element method for shell analysis, Computer Methods in Applied Mechanics and Engineering 198 (2008) 165-177.

[5] J. Sladek, V. Sladek, C. Zhang, P. Solek, Static and dynamic analysis of shallow shells with functionally graded and orthotropic material properties, Mechanics of Advanced Materials and Structures 15 (2008) 142-156.

[6] D. Benson, Y. Bazilevs, M.-C. Hsu, T. Hughes, A large deformation, rotation-free, isogeometric shell, Computer Methods in Applied Mechanics and Engineering 200 (2011) 1367-1378. 
[7] M. Cinefra, C. Chinosi, L. Della Croce, MITC9 shell elements based on refined theories for the analysis of isotropic cylindrical structures, Mechanics of Advanced Materials and Structures 20 (2013) 91-100.

[8] T. Sussman, K.-J. Bathe, 3D-shell elements for structures in large strains, Computers \& Structures 122 (2013) 2-12.

[9] Y. Lee, P.-S. Lee, K.-J. Bathe, The MITC3+ shell element and its performance, Computers \& Structures 138 (2014) 12-23.

[10] J. Woo, S. Meguid, Nonlinear analysis of functionally graded plates and shallow shells, International Journal of Solids and Structures 38 (2001) 7409-7421.

[11] G. Nie, Analysis of non-linear behaviour of imperfect shallow spherical shells on pasternak foundation by the asymptotic iteration method, International Journal of Pressure Vessels and Piping 80 (2003) 229-235.

[12] R. Heuer, F. Ziegler, Thermoelastic stability of layered shallow shells, International Journal of Solids and Structures 41 (2004) 2111-2120.

[13] M. Amabili, Non-linear vibrations of doubly curved shallow shells, International Journal of Non-Linear Mechanics 40 (2005) 683-710.

[14] N. D. Duc, H. Van Tung, Nonlinear response of pressure-loaded functionally graded cylindrical panels with temperature effects, Composite Structures 92 (2010) 1664-1672.

[15] A. Sofiyev, N. Kuruoglu, Buckling analysis of nonhomogeneous orthotropic thin-walled truncated conical shells in large deformation, Thin-Walled Structures 62 (2013) 131-141.

[16] J. Jiang, P. Khazaeinejad, A. Usmani, Nonlinear analysis of shell structures in fire using opensees, in: Proceedings of the 20th UK Conference of the Association for Computational Mechanics in Engineering (ACME2012). 
[17] L. H. Donnell, Beams, plates and shells, volume 8, McGraw-Hill New York, 1976.

[18] M. S. Qatu, Vibration of laminated shells and plates, Elsevier, Amsterdam., 2004.

[19] P. Khazaeinejad, A. Usmani, O. Laghrouche, Nonlinear stress analysis of plates under thermo-mechanical loads, in: Journal of Physics: Conference Series, volume 382, IOP Publishing, p. 012022.

[20] P. Khazaeinejad, A. Usmani, O. Laghrouche, An analytical study of the nonlinear thermo-mechanical behaviour of thin isotropic rectangular plates, Computers \& Structures 141 (2014) 1-8.

[21] J. Sladek, V. Sladek, P. Solek, P. Wen, S. Atluri, Thermal analysis of Reissner-Mindlin shallow shells with FGM properties by the MLPG, CMES: Computer Modeling in Engineering \& Sciences 30 (2008) 77-97. 